\title{
ON A TEST FOR THE CONVERGENCE \\ OF FOURIER SERIES
}

FU CHENG HSIANG

Suppose that $\phi(t)$ is an even and integrable function, periodic with period $2 \pi$. Let

$$
\phi(t) \sim \frac{1}{2} a_{0}+\sum_{\nu=1}^{\infty} a_{\nu} \cos \nu t .
$$

Wang $^{1}$ has established the following two theorems.

THEOREM A. If

$$
\begin{aligned}
\int_{0}^{t} \phi(u) d u & =o\left(\frac{t}{\log (1 / t)}\right) \quad(t \rightarrow+0) \\
a_{n} & >-K \frac{\log n}{n}
\end{aligned}
$$

for some $K>0$, then the series $\sum a_{n}$ converges.

THEOREM B. There exists an even function $\phi(t)$, satisfying (i), whose Fourier series diverges at $t=0$, while

$$
a_{n}=o\left(\frac{(\log n)^{2}}{n}\right) .
$$

The problem of bridging the gap between Theorems $A$ and $B$ is still unsolved. In this note, we remove this gap by proving the following

THEOREM. There exists an even function $\phi(t)$, satisfying (i), whose Fourier series diverges at $t=0$, while

$$
a_{n}=o\left(\frac{(\log n)^{1+\eta}}{n}\right),
$$

where $\eta$ is any preassigned positive value.

Proof. We choose three sequences of positive values $\left(\lambda_{i}\right),\left(\alpha_{i}\right)$ and $\left(c_{i}\right)$ such that

Received by the editors November 14, 1955.

1 Proc. London Math. Soc. (2) vol. 47 (1942) p. 308. 


$$
\begin{aligned}
0 & <\lambda_{1}<\lambda_{2}<\cdots<\lambda_{n} \rightarrow \infty ;^{2} \\
\alpha_{i} & =\log \lambda_{i} / \lambda_{i} \\
c_{i} & =\left(\log \alpha_{i-1} / \alpha\right)^{-1 /(1+\eta) .} \quad(i \geqq 1), \alpha_{0}=\pi ;
\end{aligned}
$$

And define an even function:

$$
\phi(t)=c_{i} \sin \lambda_{i} t \quad\left(\alpha_{i}<t \leqq \alpha_{i-1} ; i=1,2,3, \cdots\right) .
$$

Then, $\phi(t)$, being bounded, is integrable in the Lebesgue sense.

By a number of direct calculations, ${ }^{3}$ we can arrive at the following results:

$$
\frac{1}{t} \log \frac{1}{t} \int_{0}^{t} \phi(u) d u=\frac{1}{\alpha_{n}} \log \underset{\alpha_{n}}{=} O\left(\sum_{i \geqq n} \frac{c_{i}}{\lambda_{i}}\right) \text { for } \alpha_{n}<t \leqq \alpha_{n-1} .
$$

Let the $\lambda_{n}$ th partial sum of the Fourier series of $\phi(t)$ at $t=0$ be $S_{\lambda_{n}}$, then

$$
\begin{aligned}
S_{\lambda_{n}}= & \frac{2}{\pi} \int_{0}^{\pi} \phi(t) \frac{\sin \lambda_{n} t}{t} d t+o(1) \\
= & \frac{2}{\pi}\left(\sum_{i<n}+\sum_{i>n}\right) c_{i} \int_{\alpha_{i}}^{\alpha_{i}-1} \frac{\sin \lambda_{i} t \sin \lambda_{n} t}{t} d t \\
& +\frac{2 c_{n}}{\pi} \int_{\alpha_{n}}^{\alpha_{n-1}} \frac{\sin ^{2} \lambda_{n} t}{t} d t+o(1) \\
= & \frac{2}{\pi}\left(\Sigma_{1}+\Sigma_{2}+\Sigma_{3}\right)+o(1) .
\end{aligned}
$$

We have

$$
\begin{aligned}
\Sigma_{1} & =\sum_{i<n} c_{i} \int_{\alpha_{i}}^{\alpha_{i}-1} \frac{\sin \lambda_{i} t \sin \lambda_{n} t}{t} d t \\
& =O\left(\sum_{i<n} \frac{c_{i}}{\alpha_{i}\left(\lambda_{n}-\lambda_{i}\right)}\right)=\frac{1}{\lambda_{n}} O\left(\sum_{i<n} \frac{c_{i}}{\alpha_{i}}\right),
\end{aligned}
$$

if $\lambda_{n-1} / \lambda_{n}<k<1$ for every $n$.

$$
\Sigma_{2}=\sum_{i>n} c_{i} \int_{\alpha_{i}}^{\alpha_{i-1}} \frac{\sin \lambda_{i} t \sin \lambda_{n} t}{t} d t=\lambda_{n} O\left(\sum_{i>n} \frac{c_{i} \alpha_{i-1}}{\lambda_{i} \alpha_{i}}\right),
$$

${ }^{2}$ We take the sequence $\left(\lambda_{i}\right)$ in such a manner that $\alpha_{i}<\alpha_{i-1}$ for every $i$ and $c_{i}=o(1)$ as $i \rightarrow \infty$.

${ }^{3}$ For the detailed derivation of these expressions, cf. Proc. London Math. Soc. loc. cit., pp. 316-318. 
since the total variation of $\sin \lambda_{n} t$ in $\left(\alpha_{i}, \alpha_{i-1}\right)$ is $O\left(\alpha_{i-1} \lambda_{n}\right)$.

$$
\Sigma_{3}=c_{n} \int_{\alpha_{n}}^{\alpha_{n}-1} \frac{\sin ^{2} \lambda_{n} t}{t} d t=\frac{c_{n}}{2} \log \frac{\alpha_{n-1}}{\alpha_{n}}+O\left(\frac{c_{n}}{\alpha_{n} \lambda_{n}}\right) .
$$

Finally, we have

$$
\begin{aligned}
a_{n} & =\frac{2}{\pi} \int_{0}^{\pi} \phi(t) \cos n t d t=\frac{2}{\pi}\left(\sum_{i \leqq \rho_{n}}+\sum_{i>\rho_{n}}\right) c_{i} \int_{\alpha_{i}}^{\alpha_{i}-1} \sin \lambda_{i} t \cos n t d t \\
& =\frac{2}{\pi}\left(\sum_{i \leqq \rho_{n}} J_{i}+\sum_{i>\rho_{n}} J_{i}\right)
\end{aligned}
$$

say, where $\left(\rho_{n}\right)$ is a rapidly increasing sequence of positive integers.

Now,

$$
\sum_{i \leq \rho_{n}} J_{i}=\sum_{i \leqq \rho_{n}} c_{i} \int_{\alpha_{i}}^{\alpha_{i}-1} \sin \lambda_{i} t \cos n t d t=\frac{1}{n} O\left(\sum_{i \leqq \rho_{n}} c_{i} \lambda_{i} \alpha_{i-1}\right) .
$$

On the other hand,

$$
\sum_{i>\rho_{n}} J_{i}=O\left(\sum_{i>\rho_{n}} \frac{c_{i}}{\left|\lambda_{i}-n\right|}\right)
$$

In order to establish the theorem, we need to make the value in (1), $o(1), \Sigma_{1}+\Sigma_{2}=o(1), \Sigma_{3}>1$ and the values of the expressions in (5) and (6) are both $o\left((\log n)^{1+\eta} / n\right)$.

We take $\lambda_{i}=[((i+1) !) \eta]$. Then

$$
\frac{1}{t} \log \frac{1}{t} \int_{0}^{t} \phi(u) d u=((n+1) !)^{\eta} o\left(\sum_{i \geqq n} \frac{1}{((i+1) !) \eta}\right)=o(1)
$$

as $t \rightarrow+o$. And

$$
\begin{aligned}
\Sigma_{1} & =\frac{1}{((n+1) !)^{\eta}} O\left(\sum_{i<n} \frac{((i+1) !)^{\eta}}{\log (i+1) !}\right) \\
& =\frac{1}{((n+1) !)^{\eta}} O\left(\frac{(n !)^{\eta}}{\log n}\right)=o(1) . \\
\Sigma_{2} & =((n+1) !)^{\eta} o\left(\sum_{i>n} \frac{1}{(i !) \eta}\right)=o(1) . \\
\Sigma_{3} & \cong \frac{\eta}{2(\log (n+1))^{1 /(1+\eta)}} \log \frac{(n+1) !}{n !}+o(1) \\
& =\frac{\eta}{2}(\log (n+1))^{\eta /(1+\eta)}+o(1)>1 .
\end{aligned}
$$


This shows that the Fourier series of $\phi(t)$ diverges at $t=0$.

Moreover,

$$
\begin{aligned}
& \sum_{i \leqq \rho_{n}} J_{i}=\frac{1}{n} o\left(\sum_{i \leqq \rho_{n}}\left((i+1 !)^{\eta} \frac{\log i !}{(i !)^{\eta}}\right)=\frac{1}{n} o\left(\rho_{n}^{2+\eta} \log \rho_{n}\right),\right. \\
& \sum_{i>\rho_{n}} J_{i}=o\left(\sum_{i>\rho_{n}} \frac{1}{((i+1) !)^{\eta}-n}\right)=o\left(\sum_{i>\rho_{n}} \frac{1}{((i+1) !)^{\eta}-(i !)^{\eta}}\right)
\end{aligned}
$$

since $(i !)^{\eta} \geqq \lambda_{i-1} \geqq \lambda_{\rho_{n}}>n$. Therefore,

$$
\sum_{i>\rho_{n}} J_{i}=o\left(\frac{1}{\left(\rho_{n} !\right)^{\eta}}\right)=o\left(\frac{e^{\eta \rho_{n}}}{\rho_{n}^{\eta\left(\rho_{n}+1 / 2\right)}}\right)
$$

by Stirling's formula

$$
e^{n} n ! \cong(2 \pi)^{1 / 2} n^{n+1 / 2}
$$

Now, if we choose a rapidly increasing sequence of positive integers $\left(n_{i}\right)$ such as $n_{i}=((i+1) !)$ ! and define another function $\phi^{*}(t)$ from $\phi(t)$ as follows:

$$
\phi^{*}(t)=c_{i}^{*} \sin \lambda_{i}^{*} t \quad\left(\alpha_{i}^{*}<t \leqq \alpha_{i-1}^{*}\right),
$$

where $\lambda_{i}{ }^{*}=\lambda_{i}, \alpha_{i}{ }^{*}=\alpha_{i}$ and $c_{\nu}{ }^{*}=c_{n_{i}}$ if $\nu=n_{i}$ for a certain $i$; and $c_{\nu}{ }^{*}=0$ if $\nu \neq n_{i}$ for every $i$, under this circumstance, the corresponding condition (i) of the theorem is still satisfied and the Fourier series of $\phi^{*}(t)$ still diverges at $t=0$ as before. But, by this method of choice of the sequence $\left(n_{i}\right)$, we can easily find that the corresponding

$$
\sum_{i \leqq \rho_{n}} J_{i}^{*}=\frac{1}{n} o\left(\rho_{n}^{1+\eta} \log \rho_{n}\right) .
$$

Taking $\rho_{n}=\left[\log n\left(\log ^{2} n\right)^{-1 /(1+\eta)}\right]$, we obtain

$$
\sum_{i \leq p_{n}} J_{i}^{*}=o\left(\frac{(\log n)^{1+\eta}}{n}\right),
$$

also,

$$
\sum_{i>\rho_{n}} J_{i}^{*}=o\left(\frac{(\log n)^{1+\eta}}{n}\right) .
$$

Thus, $a_{n}^{*}=o\left((\log n)^{1+\eta / n}\right)$. This completes the proof of the theorem. 